PROCEEDINGS OF THE AMERICAN MATHEMATICAL SOCIETY

Volume 124, Number 9, September 1996

\title{
COUNTABLE NETWORK WEIGHT AND MULTIPLICATION OF BOREL SETS
}

\author{
D. H. FREMLIN, R. A. JOHNSON, AND E. WAJCH
}

(Communicated by Franklin D. Tall)

\begin{abstract}
A space $X$ Borel multiplies with a space $Y$ if each Borel set of $X \times Y$ is a member of the $\sigma$-algebra in $X \times Y$ generated by Borel rectangles. We show that a regular space $X$ Borel multiplies with every regular space if and only if $X$ has a countable network. We give an example of a Hausdorff space with a countable network which fails to Borel multiply with any non-separable metric space. In passing, we obtain a characterization of those spaces which Borel multiply with the space of countable ordinals, and an internal necessary and sufficient condition for $X$ to Borel multiply with every metric space.
\end{abstract}

\section{INTRODUCTION}

For a topological space $X$, denote by $\mathcal{B}(X)$ the collection of Borel sets of $X$, i.e. the smallest $\sigma$-algebra containing the open sets of $X$. If $X$ and $Y$ are topological spaces, then $\mathcal{B}(X) \otimes \mathcal{B}(Y)$ denotes the smallest $\sigma$-algebra in $X \times Y$ containing all sets of the form $E \times F$ where $E$ and $F$ are Borel sets of $X$ and $Y$, respectively. The inclusion $\mathcal{B}(X) \otimes \mathcal{B}(Y) \subseteq \mathcal{B}(X \times Y)$ always holds; if $\mathcal{B}(X) \otimes \mathcal{B}(Y)=\mathcal{B}(X \times Y)$, we say that $X$ Borel multiplies with $Y$. A space $X$ will be called a universal Borel multiplier if $X$ Borel multiplies with every space. Considerable attention has been devoted to the Borel multiplication of discrete spaces (cf. e.g. [1], [8], [9], [12], [13] $\&[15])$; however, the following natural problem does not seem to have been posed explicitly:

Problem. Give an internal characterization of universal Borel multipliers.

It is well known that any second countable space $X$ Borel multiplies with every space (cf. [7, Thm. 8]). It is easy to observe that if $X$ is regular, the assumption of second countability can be weakened to the requirement that $X$ be of countable network weight. We shall prove that countable network weight is also a necessary condition for a regular space to be a universal Borel multiplier. To this end, we shall show first that if a space $X$ Borel multiplies with the space $\omega_{1}$ of countable ordinals equipped with the order topology, then $X$ is hereditarily Lindelöf. Further, we shall observe that if a regular Hausdorff hereditarily Lindelöf space $X$ Borel multiplies with any one of its Hausdorff compactifications, then $X$ has a countable network. The above-mentioned results imply that a regular (not necessarily Hausdorff) space

Received by the editors February 21, 1995.

1991 Mathematics Subject Classification. Primary 54H05, $28 \mathrm{~A} 05$.

Key words and phrases. Borel set, product $\sigma$-algebra, countable network, hereditary separability, hereditary Lindelöf property, metric space, countable ordinals. 
$X$ is a universal Borel multiplier if and only if $X$ is of countable network weight. Since a useful characterization of spaces Borel multiplying with $\omega_{1}$ seems to be interesting in itself, we shall take the opportunity to prove that $X$ Borel multiplies with $\omega_{1}$ if and only if $X$ is a hereditarily separable hereditarily Lindelöf space which Borel multiplies with the discrete space of size $\omega_{1}$. We shall find an internal necessary and sufficient condition for $X$ to Borel multiply with every metric space. Finally, we shall give an example of a Hausdorff space of countable network weight which fails to Borel multiply with the discrete space of size $\omega_{1}$.

We shall use $h d(X)$ and $h l(X)$ to denote, respectively, the hereditary density and the hereditary Lindelöf degree of $X$. Basic facts concerning the cardinal functions considered here can be found, for instance, in [2] and [10]. The real line with the usual topology induced by the metric $|x-y|$ will be denoted by $\mathbb{R}$. The symbol $\mathcal{P}(X)$ will stand for the collection of all subsets of $X$. For a cardinal $\kappa, D(\kappa)$ will denote the discrete space of size $\kappa$.

\section{THE RESUlTS}

For completeness, let us begin with the following

1. Proposition. Let $X$ and $Y$ be topological spaces. If $X$ has a countable network consisting of Borel sets, then $\mathcal{B}(X) \otimes \mathcal{B}(Y)=\mathcal{B}(X \times Y)$.

Proof. Suppose that $\left\langle E_{n}\right\rangle_{n<\omega}$ is a countable collection of Borel sets of $X$ which serves as a network for $X$. To check that $\mathcal{B}(X \times Y) \subseteq \mathcal{B}(X) \otimes \mathcal{B}(Y)$, it suffices to observe that if $W \subseteq X \times Y$ is open, then $W=\bigcup_{n<\omega}\left(E_{n} \times V_{n}\right)$ where $V_{n}=\bigcup\{V: V$ is open in $Y$ and $\left.E_{n} \times V \subseteq W\right\}$ for $n<\omega$.

2. Corollary. A regular space of countable network weight is a universal Borel multiplier.

If $M \subseteq X \times Y$, the horizontal section of $M$ at $y \in Y$ is defined by $M^{-1}[y]=$ $\{x \in X:(x, y) \in M\}$, and the vertical section of $M$ at $x \in X$ is given by $M[x]=$ $\{y \in Y:(x, y) \in M\}$.

3. Lemma. Let $\mathcal{A}_{X}$ be a $\sigma$-algebra of subsets of $X$, let $\mathcal{I}$ be a $\sigma$-ideal of subsets of $Y$, and let $\mathcal{A}_{Y}$ be the smallest $\sigma$-algebra of subsets of $Y$ which contains $\mathcal{I}$. That is, $\mathcal{A}_{Y}=\{A \subseteq Y: A \in \mathcal{I}$ or $Y \backslash A \in \mathcal{I}\}$. Then, for each $M \in \mathcal{A}_{X} \otimes \mathcal{A}_{Y}$, we have

$$
\bigcup\{M[x]: M[x] \in \mathcal{I}\} \in \mathcal{I} \quad \text { and } \quad \bigcup\{Y \backslash M[x]: M[x] \notin \mathcal{I}\} \in \mathcal{I} .
$$

Proof. Let $\mathcal{M}$ be the family of all the sets $M \subseteq X \times Y$ which have the property that there exists $L \in \mathcal{I}$ such that, for each $x \in X, M[x] \subseteq L$ if $M[x] \in \mathcal{I}$, and $Y \backslash M[x] \subseteq L$ if $M[x] \notin \mathcal{I}$. Then $\mathcal{M}$ is stable under complements; indeed, the same $L$ will work for a set $M$ and its complement. To show that $\mathcal{M}$ is closed under countable unions, suppose that $\left\langle M_{n}\right\rangle_{n<\omega}$ is a sequence of members of $\mathcal{M}$. For any $n<\omega$, choose $L_{n} \in \mathcal{I}$ such that, for each $x \in X, M_{n}[x] \subseteq L_{n}$ if $M_{n}[x] \in \mathcal{I}$, and $Y \backslash M_{n}[x] \subseteq L_{n}$ if $M_{n}[x] \notin \mathcal{I}$. Put $L=\bigcup_{n<\omega} L_{n}$. Obviously, $L \in \mathcal{I}$. If $\left(\bigcup_{n<\omega} M_{n}\right)[x] \in \mathcal{I}$, then $M_{n}[x] \in \mathcal{I}$ for any $n<\omega$, so that $\bigcup_{n<\omega} M_{n}[x] \subseteq L$. On the other hand, if $\left(\bigcup_{n<\omega} M_{n}\right)[x] \notin \mathcal{I}$, then there exists $m<\omega$ such that $M_{m}[x] \notin \mathcal{I}$; for this $m$ we have $Y \backslash M_{m}[x] \subseteq L$, which implies that $Y \backslash\left(\bigcup_{n<\omega} M_{n}\right)[x] \subseteq L$. We have therefore shown that $\mathcal{M}$ is a $\sigma$-algebra. Clearly, $\mathcal{M}$ contains each rectangle of the form $E \times F$ where $E \in \mathcal{A}_{X}$ and $F \in \mathcal{A}_{Y}$ (simply, let $L=F$ if $F \in \mathcal{I}$, and $L=Y \backslash F$ otherwise). Thus $\mathcal{M}$ contains $\mathcal{A}_{X} \otimes \mathcal{A}_{Y}$ and the result follows. 
4. Theorem. A topological space $X$ Borel multiplies with $\omega_{1}$ if and only if $X$ is a hereditarily separable hereditarily Lindelöf space which Borel multiplies with $D\left(\omega_{1}\right)$.

Proof. Necessity. Assume that $\mathcal{B}(X) \otimes \mathcal{B}\left(\omega_{1}\right)=\mathcal{B}\left(X \times \omega_{1}\right)$. By [8, Lemma 1.1], it is obvious that $X$ Borel multiplies with $D\left(\omega_{1}\right)$. Let $\mu$ be the Dieudonné measure on $\omega_{1}$ (cf. $\left[3,5.5\right.$, p. 974]). Then $\mathcal{B}\left(\omega_{1}\right)$ is contained in the $\sigma$-algebra generated by the $\sigma$-ideal $\mathcal{I}=\left\{A \subseteq \omega_{1}\right.$ : there is $B \in \mathcal{B}\left(\omega_{1}\right)$ with $A \subseteq B$ and $\left.\mu(B)=0\right\}$. Suppose, if possible, that $h d(X)>\omega$. There exists a transfinite sequence $\left\langle x_{\xi}\right\rangle_{\xi<\omega_{1}}$ of points of $X$ such that $x_{\xi} \notin \operatorname{cl}\left\{x_{\eta}: \eta<\xi\right\}$ for any $\xi<\omega_{1}$. Put

$$
W=\bigcup_{\xi<\omega_{1}}\left(\left(X \backslash \operatorname{cl}\left\{x_{\eta}: \eta<\xi\right\}\right) \times \xi\right) .
$$

The set $W$ being open in $X \times \omega_{1}$, it follows from Lemma 3 that $\mu(\bigcup\{W[x]$ : $\mu(W[x])=0\})=0$. On the other hand, since $\mu(\xi)=0$ and $W\left[x_{\xi}\right]=\xi$ for any $\xi<$ $\omega_{1}$, we have $\bigcup\{W[x]: \mu(W[x])=0\}=\omega_{1}$; hence $\mu(\bigcup\{W[x]: \mu(W[x])=0\})=1$. The contradiction obtained shows that $h d(X) \leq \omega$.

Now, suppose that $h l(X)>\omega$. There exists a collection $\left\langle V_{\xi}\right\rangle_{\xi<\omega_{1}}$ of open subsets of $X$ such that $V_{\xi} \subseteq V_{\eta}$ and $V_{\xi} \neq V=\bigcup_{\tau<\omega_{1}} V_{\tau}$ for any $\xi<\eta<\omega_{1}$. Let

$$
U=\bigcup_{\xi<\omega_{1}}\left(V_{\xi} \times\left[\xi+1, \omega_{1}\right)\right)
$$

For any $x \in V$, let $\xi_{x}$ be the first ordinal such that $x \in V_{\xi_{x}}$. Then $U[x]=\left[\xi_{x}+1, \omega_{1}\right)$ for $x \in V$, hence $\bigcup\left\{\omega_{1} \backslash U[x]: \mu(U[x])=1\right\}=\omega_{1}$. Since $U$ is open in $X \times \omega_{1}$, it follows from Lemma 3 that $\mu\left(\bigcup\left\{\omega_{1} \backslash U[x]: \mu(U[x])=1\right\}\right)=0$, which is absurd. Therefore $h l(X) \leq \omega$.

Sufficiency. Given a closed set $F \subseteq X \times \omega_{1}$, put

$$
A=\{x \in X: F[x] \text { is unbounded }\} .
$$

Consider any $x \in X \backslash A$. We shall show that there exist an open neighbourhood $U_{x}$ of $x$ and an ordinal $\gamma_{x}<\omega_{1}$, such that

$$
\left(U_{x} \times\left[\gamma_{x}, \omega_{1}\right)\right) \cap F=\emptyset .
$$

To this end, choose $\eta_{x}<\omega_{1}$ with $F[x] \subseteq \eta_{x}$. If $\eta_{x}<\alpha<\omega_{1}$, then $\left(\{x\} \times\left[\eta_{x}, \alpha\right]\right) \cap$ $F=\emptyset$; hence, by the compactness of $\left[\eta_{x}, \alpha\right]$, there exists an open neighbourhood $G_{\alpha}$ of $x$ such that $\left(G_{\alpha} \times\left[\eta_{x}, \alpha\right]\right) \cap F=\emptyset$. Let $U_{x}=\bigcap_{\alpha>\eta_{x}} \bigcup_{\beta>\alpha} G_{\beta}$. Since $h d(X) \leq \omega$, there exists a countable $B \subseteq X \backslash U_{x}$ such that cl $B=X \backslash U_{x}$. For any $y \in B$, we can find $\alpha_{y}>\eta_{x}$ with $y \in \bigcap_{\beta>\alpha_{y}}\left(X \backslash G_{\beta}\right)$. Let $\gamma_{x}=\sup _{y \in B} \alpha_{y}$. Then $B \subseteq \bigcap_{\beta>\gamma_{x}}\left(X \backslash G_{\beta}\right)$, which implies that $X \backslash U_{x}=\bigcap_{\beta>\gamma_{x}}\left(X \backslash G_{\beta}\right)$. In consequence, $U_{x}$ is an open neighbourhood of $x$. It is easily seen that $U_{x} \times\left[\gamma_{x}, \omega_{1}\right)$ does not meet $F$. Obviously, $U_{x} \subseteq X \backslash A$, which means that $A$ is closed. Since $h l(X) \leq \omega$, there exists a countable $C \subseteq X \backslash A$ such that $\bigcup_{x \in X \backslash A} U_{x}=\bigcup_{x \in C} U_{x}$. For $\zeta_{0}=\sup _{x \in C} \gamma_{x}$, we have

$$
\left((X \backslash A) \times\left[\zeta_{0}, \omega_{1}\right)\right) \cap F=\emptyset .
$$

As $h d(X) \leq \omega$, there exists a countable $D \subseteq A$ such that $\operatorname{cl} D=A$. Put

$$
H=\bigcap_{x \in D}\left(F[x] \cap\left[\zeta_{0}, \omega_{1}\right)\right) .
$$

Let us observe that if $\xi \in H$, then $D \subseteq F^{-1}[\xi] \subseteq A$, so that $F^{-1}[\xi]=A$ for any $\xi \in H$. This yields that $F \cap(X \times H)=A \times H \in \mathcal{B}(X) \otimes \mathcal{B}\left(\omega_{1}\right)$. Further, since the 
collection of closed unbounded subsets of $\omega_{1}$ is stable under countable intersections, the set $H$ is closed and unbounded. Accordingly $\omega_{1} \backslash H$ is a metrizable open subspace of $\omega_{1}$. By Theorem 2.5 of [8], $X$ Borel multiplies with $\omega_{1} \backslash H$. Therefore $F \cap\left(X \times\left(\omega_{1} \backslash H\right)\right) \in \mathcal{B}(X) \otimes \mathcal{B}\left(\omega_{1}\right)$, which completes the proof.

A satisfactory solution of the problem of when a space can Borel multiply with $D\left(\omega_{1}\right)$ is unknown. Even the answer to the question whether $\mathcal{B}(D(\kappa)) \otimes \mathcal{B}\left(D\left(\omega_{1}\right)\right)=$ $\mathcal{B}\left(D(\kappa) \times D\left(\omega_{1}\right)\right)$ for $\kappa \leq 2^{\omega}$ depends on one's set theory (cf. e.g. [1] \& [9]). However, with Theorem 4 in hand it is easy to check that, for instance, the Sorgenfrey line Borel multiplies with $\omega_{1}$. To see this, let us give an internal characterization of those spaces which Borel multiply with every metric space.

Given a collection $\mathcal{G}$ of subsets of $X$, denote by $[\mathcal{G}]_{\sigma}$ and $[\mathcal{G}]_{c}$ the collections of, respectively, countable unions and complements of members of $\mathcal{G}$. Put $\mathcal{B}_{0}(\mathcal{G})=$ $[\mathcal{G}]_{\sigma} \cup\{\emptyset\}$ and, for any non-zero ordinal $\alpha<\omega_{1}$, define

$$
\mathcal{B}_{\alpha}(\mathcal{G})= \begin{cases}{\left[\bigcup_{\eta<\alpha} \mathcal{B}_{\eta}(\mathcal{G})\right]_{\sigma}} & \text { when } \alpha \text { is even, } \\ {\left[\bigcup_{\eta<\alpha} \mathcal{B}_{\eta}(\mathcal{G})\right]_{c}} & \text { when } \alpha \text { is odd }\end{cases}
$$

Then $\bigcup_{\alpha<\omega_{1}} \mathcal{B}_{\alpha}(\mathcal{G})$ equals the smallest $\sigma$-algebra containing $\mathcal{G}$.

5. Theorem. A topological space $X$ Borel multiplies with every metric space if and only if there exist a countable ordinal $\alpha$ and a countable collection $\mathcal{G}$ of open subsets of $X$, such that any open set in $X$ lies in $\mathcal{B}_{\alpha}(\mathcal{G})$.

Proof. Necessity. Let $\left\{W_{y}: y \in Y\right\}$ be the collection of all open subsets of $X$. Give $Y$ the discrete topology and put $W=\bigcup_{y \in Y}\left(W_{y} \times\{y\}\right)$. If $W \in \mathcal{B}(X) \otimes \mathcal{B}(Y)$, then, in view of [5, Exercise 59.2, p. 261], there exist countable collections $\mathcal{G}$ of open subsets of $X$ and $\mathcal{A}$ of subsets of $Y$, such that $W$ lies in the smallest $\sigma$-algebra which contains all rectangles of the form $G \times A$ where $G \in \mathcal{G}$ and $A \in \mathcal{A}$. We can find a countable ordinal $\alpha$ such that $W \in \mathcal{B}_{\alpha}(\{G \times A: G \in \mathcal{G} \quad \& \quad A \in \mathcal{A}\})$. It is readily seen that $\left\{W_{y}: y \in Y\right\} \subseteq \mathcal{B}_{\alpha}(\mathcal{G})$.

Sufficiency. Suppose now that we are given a countable ordinal $\alpha$ and a countable collection $\mathcal{G}$ of open subsets of $X$, such that any open set in $X$ lies in $\mathcal{B}_{\alpha}(\mathcal{G})$. Let $Y$ be any discrete space. One easily checks that if $W \subseteq X \times Y$ is such that $W^{-1}[y] \in$ $\mathcal{B}_{0}(\mathcal{G})$ for any $y \in Y$, then $W \in \mathcal{B}(X) \otimes \mathcal{B}(Y)$. Suppose that we have already shown that if $\xi<\alpha$, and $W \subseteq X \times Y$ is such that $W^{-1}[y] \in \mathcal{B}_{\xi}(\mathcal{G})$ for any $y \in Y$, then $W \in \mathcal{B}(X) \otimes \mathcal{B}(Y)$. Consider any set $U \subseteq X \times Y$ such that $U^{-1}[y] \in \mathcal{B}_{\alpha}(\mathcal{G})$ for any $y \in Y$. Let $\alpha$ be even. For any $y \in Y$, choose a sequence $\left\langle\xi_{n}(y)\right\rangle_{n<\omega}$ of ordinals $<\alpha$ and sets $A_{n}(y) \in \mathcal{B}_{\xi_{n}(y)}(\mathcal{G})$, such that $U^{-1}[y]=\bigcup_{n<\omega} A_{n}(y)$. Let $C_{n, \xi}=\left\{y \in Y: \xi_{n}(y)=\xi\right\}$ and $U_{n, \xi}=\bigcup\left\{A_{n}(y) \times\{y\}: y \in C_{n, \xi}\right\}$ for $\xi<\alpha$ and $n<\omega$. Under the inductive assumption, $U_{n, \xi} \in \mathcal{B}(X) \otimes \mathcal{B}(Y)$ for any $\xi<\alpha$ and $n<\omega$. Since $U=\bigcup_{\xi<\alpha} \bigcup_{n<\omega} U_{n, \xi}$, we have that $U \in \mathcal{B}(X) \otimes \mathcal{B}(Y)$. If $\alpha$ is odd, we can use similar arguments to show that $(X \times Y) \backslash U \in \mathcal{B}(X) \otimes \mathcal{B}(Y)$. In consequence, we obtain that $X$ Borel multiplies with $Y$. By Theorem 2.5 of [8], $X$ Borel multiplies with every metric space.

6. Corollary. The Sorgenfrey line Borel multiplies with every metric space and with $\omega_{1}$.

Obviously, the Sorgenfrey line does not Borel multiply with itself for it contains only $2^{\omega}$ Borel sets, while its square contains $2^{2^{\omega}}$ Borel sets. 
Let us come back to the question whether a universal Borel multiplier is of countable network weight.

7. Lemma. If $\mathcal{E}$ is a collection of subsets of a set $Y$ and if $F$ lies in the smallest $\sigma$-algebra containing $\mathcal{E}$, then

$$
(F \times(Y \backslash F)) \cup((Y \backslash F) \times F) \subseteq \bigcup_{E \in \mathcal{E}}((E \times(Y \backslash E)) \cup((Y \backslash E) \times E)) .
$$

Proof. It is enough to observe that $\mathcal{M}=\{M \subseteq Y:(M \times(Y \backslash M)) \cup((Y \backslash M) \times M) \subseteq$ $\left.\bigcup_{E \in \mathcal{E}}((E \times(Y \backslash E)) \cup((Y \backslash E) \times E))\right\}$ is a $\sigma$-algebra which contains $\mathcal{E}$.

8. Proposition. Let $X$ be a hereditarily Lindelöf regular Hausdorff space. If there exists a Hausdorff compactification $\alpha X$ of $X$ such that $\mathcal{B}(X \times \alpha X)=\mathcal{B}(X) \otimes \mathcal{B}(\alpha X)$, then $X$ has a countable network.

Proof. The diagonal $\Delta$ of $X$ is a closed subset of $X \times \alpha X$; hence, since $\Delta \in$ $\mathcal{B}(X) \otimes \mathcal{B}(\alpha X)$, there exists a countable collection $\mathcal{G}$ of open subsets of $\alpha X$ such that $\Delta$ lies in the smallest $\sigma$-algebra containing all sets of the form $(G \cap X) \times H$ where $G, H \in \mathcal{G}$ (cf. [5, Exercise 59.2, p. 261]). It follows from Lemma 7 that, for any $x \in X$ and $y \in \alpha X$ with $x \neq y$, there exists $G \in \mathcal{G}$ such that exactly one of the points $x, y$ lies in $G$. Since $X$ is regular and hereditarily Lindelöf, for any $G \in \mathcal{G}$, there exists a countable collection $\mathcal{F}_{G}$ of closed subsets of $\alpha X$ such that $G \cap X \subseteq \bigcup_{F \in \mathcal{F}_{G}} F \subseteq G$. Put $\mathcal{F}=\{\alpha X \backslash G: G \in \mathcal{G}\} \cup \bigcup_{G \in \mathcal{G}} \mathcal{F}_{G}$. The collection $\mathcal{F}$ is countable and has the property that, for any $x \in X$ and $y \in \alpha X$ with $x \neq y$, there exists $F \in \mathcal{F}$ such that $x \in F$ and $y \notin F$. This implies that if $U$ is an open subset of $\alpha X$ and if $x \in X \cap U$, then the collection $\{\alpha X \backslash F: F \in \mathcal{F} \quad \& \quad x \in F\}$ is an open cover of $\alpha X \backslash U$. There exists a finite subcollection $\mathcal{F}(x)$ of $\mathcal{F}$ such that $x \in \bigcap_{F \in \mathcal{F}(x)} F$ and $\alpha X \backslash U \subseteq \bigcup_{F \in \mathcal{F}(x)}(\alpha X \backslash F)$. This shows that the collection of all finite intersections of members of $\{F \cap X: F \in \mathcal{F}\}$ is a countable network for $X$.

Since the discrete space $D\left(\omega_{1}\right)$ Borel multiplies with itself (cf. [9, Thm. 12.5(ii)] or $[12$, Thm. 2]), it can serve as an example of a space of uncountable network weight which Borel multiplies with its one-point compactification (cf. [8, Lemma $1.2])$.

Now, we are in a position to state our main theorem.

9. Theorem. A regular space $X$ is a universal Borel multiplier if and only if $X$ has a countable network.

Proof. For $x, y \in X$, write $x \backsim y$ if and only if $x, y$ belong to just the same open sets in $X$. It is easy to check that the quotient space $X / \backsim$ Borel multiplies with $Y$ if and only if $X$ does; $X / \backsim$ has a countable network if and only if $X$ has; further, $X / \backsim$ is regular if and only if $X$ is. Obviously, if $X$ is regular, the space $X / \backsim$ is Hausdorff. Hence, in view of Corollary 2, it suffices to prove that a regular Hausdorff universal Borel multiplier is of countable network weight. But this follows directly from Theorem 4, Proposition 8 and from the fact that every regular Hausdorff Lindelöf space is Tychonoff and, in consequence, it has a Hausdorff compactification.

Remarks. Let us observe that the collection $\mathcal{F}$ obtained in the proof of Proposition 8 witnesses that $X$ is a $\Sigma$-space; therefore, in view of Theorem 4 and [8, Lemma 1.1], the "only if" part of Theorem 9 can be deduced from Corollary 4.13 of [6] 
or from Theorems 4.4 and 4.5 of [4]. This was the original route to Theorem 9 , discovered with the help of A.V. Arhangel'skii.

The problem whether every Hausdorff universal Borel multiplier is of countable network weight is still open. It is easily seen that

$$
\mathcal{B}\left(\left(X_{1} \times X_{2}\right) \times Y\right)=\mathcal{B}\left(X_{1} \times X_{2}\right) \otimes \mathcal{B}(Y)
$$

whenever $\mathcal{B}\left(X_{1} \times\left(X_{2} \times Y\right)\right)=\mathcal{B}\left(X_{1}\right) \otimes \mathcal{B}\left(X_{2} \times Y\right)$ and $\mathcal{B}\left(X_{2} \times Y\right)=\mathcal{B}\left(X_{2}\right) \otimes \mathcal{B}(Y)$. This, along with Lemma 1.5 of [8], yields that if $\left\langle X_{n}\right\rangle_{n<\omega}$ is a sequence of topological spaces each one of which is a universal Borel multiplier, then $\prod_{n<\omega} X_{n}$ is a universal Borel multiplier. Of course, this remark is of no interest unless there are universal Borel multipliers without countable networks.

Let us finish by giving the promised example of a Hausdorff space of countable network weight which does not Borel multiply with the discrete space of size $\omega_{1}$.

10. Example. Give the set $X=\omega_{1}$ the cofinite topology. Let $\left\langle D_{\xi}\right\rangle_{\xi<\omega_{1}}$ be a partition of $\mathbb{R}$ into Bernstein sets (cf. [11, Thm. 27, p. 152]) and let $T=\{(\xi, r) \in$ $\left.X \times \mathbb{R}: r \in D_{\xi}\right\}$ be regarded as a subspace of $X \times \mathbb{R}$. If $\left(\xi_{1}, r_{1}\right),\left(\xi_{2}, r_{2}\right)$ are distinct points of $T$, then $r_{1} \neq r_{2}$; hence $T$ is Hausdorff. Since both $X$ and $\mathbb{R}$ are of countable network weight, the space $T$ is of countable network weight.

Put $\mathcal{E}=\left\{E \subseteq T\right.$ : there exist $\xi<\omega_{1}$ and $H \in \mathcal{B}(\mathbb{R})$ with $E \cap\left(\left(\omega_{1} \backslash \xi\right) \times \mathbb{R}\right)=$ $\left.T \cap\left(\left(\omega_{1} \backslash \xi\right) \times H\right)\right\}$. Observe that if $\xi<\omega_{1}$ and $H \in \mathcal{B}(\mathbb{R})$ witness that $E \in \mathcal{E}$, then $\xi$ and $\mathbb{R} \backslash H$ yield that $T \backslash E \in \mathcal{E}$; if, for $n<\omega, \xi_{n}<\omega_{1}$ and $H_{n} \in \mathcal{B}(\mathbb{R})$ witness that $E_{n} \in \mathcal{E}$, then $\sup _{n<\omega} \xi_{n}$ and $\bigcup_{n<\omega} H_{n}$ show that $\bigcup_{n<\omega} E_{n} \in \mathcal{E}$. Hence $\mathcal{E}$ is closed under complements and countable unions.

Let $A \subseteq X$ be a finite set and let $G$ be an open subset of $\mathbb{R}$. Choose $\xi<\omega_{1}$ with $A \subseteq \xi$. Then $((X \backslash A) \times G) \cap T \cap\left(\left(\omega_{1} \backslash \xi\right) \times \mathbb{R}\right)=T \cap\left(\left(\omega_{1} \backslash \xi\right) \times G\right)$; hence $\mathcal{E}$ contains all members of the natural base of the topology of $T$. Since $T$ is hereditarily Lindelöf and $\mathcal{E}$ is closed under countable unions, $\mathcal{E}$ contains all open subsets of $T$. In consequence, $\mathcal{B}(T) \subseteq \mathcal{E}$ because $\mathcal{E}$ is a $\sigma$-algebra.

Let $Y$ be any set. Let $\mathcal{A}$ be the collection of all sets $A \subseteq T \times Y$ such that there is a $\xi<\omega_{1}$ such that, for each $y \in Y$, there exists a Borel set $H \subseteq \mathbb{R}$ with $\{t \in T:(t, y) \in A\} \cap\left(\left(\omega_{1} \backslash \xi\right) \times \mathbb{R}\right)=T \cap\left(\left(\omega_{1} \backslash \xi\right) \times H\right)$. It is easily seen that $\mathcal{A}$ is a $\sigma$-algebra. Since $\mathcal{B}(T) \subseteq \mathcal{E}, \mathcal{A}$ contains all the sets $E \times F$ with $E \in \mathcal{B}(T)$ and $F \subseteq Y$. Hence $\mathcal{B}(T) \otimes \mathcal{P}(Y) \subseteq \mathcal{A}$.

Now, let $Y=\omega_{1}$ be considered with the discrete topology. Put

$$
\left.W=\bigcup_{\xi<\omega_{1}}(((X \backslash\{\xi\}) \times \mathbb{R}) \cap T) \times\{\xi\}\right) .
$$

Then $W$ is open in $T \times Y$. Suppose that $W \in \mathcal{B}(T) \otimes \mathcal{B}(Y)$. Then $W \in \mathcal{A}$, thus there exist $\xi<\omega_{1}$ and a Borel set $H \subseteq \mathbb{R}$, such that $E=\{t \in T:(t, \xi) \in$ $W\} \cap\left(\left(\omega_{1} \backslash \xi\right) \times \mathbb{R}\right)=T \cap\left(\left(\omega_{1} \backslash \xi\right) \times H\right)$. Then $\emptyset=\{r \in \mathbb{R}:(\xi, r) \in E\}=H \cap D_{\xi}$, so, $H$ is countable. On the other hand, $D_{\xi+1}=\{r \in \mathbb{R}:(\xi+1, r) \in E\}=H \cap D_{\xi+1}$, so that $D_{\xi+1} \subseteq H$ and $H$ is cocountable, which is absurd. Hence $W \notin \mathcal{B}(T) \otimes \mathcal{B}(Y)$.

In view of Proposition 1 , the space $T$ cannot have a countable network consisting of Borel sets.

\section{ACKNOWLEDGEMENT}

The authors would like to thank Prof. A.V. Arhangel'skii for a most helpful conversation leading to the original proof of Theorem 9. 


\section{REFERENCES}

1. K. Ciesielski \& F. Galvin, Cylinder problem, Fund. Math. 127 (1987), 171-176. MR 89e:03079

2. R. Engelking, General Topology, Heldermann, 1989. MR 91c:54001

3. R.J. Gardner \& W.F. Pfeffer, Borel measures, Chapt. 22 of [10], 961-1043. MR 86c:28031

4. G. Gruenhage, Generalized metric spaces, Chapt. 10 of [10], 423-501. MR 86h:54038

5. P.R. Halmos, Measure Theory, Van Nostrand, 1950. MR 11:504d

6. R.E. Hodel, On a theorem of Arhangel'skii concerning Lindelöf p-spaces, Canad. J. Math. 27 (1975), 459-468. MR 51:11401

7. R.A. Johnson, On product measures and Fubini's theorem in locally compact spaces, Trans. Amer. Math. Soc. 123 (1966), 112-129. MR 33:5832

8. R.A. Johnson, E. Wajch \& W. Wilczyński, Metric spaces and multiplication of Borel sets, Rocky Mountain J. Math. 22 (1992), 1341-1347. MR 94c:54072

9. K. Kunen, Inaccessibility properties of cardinals, Ph.D. thesis, Stanford University, Palo Alto, 1968.

10. K. Kunen \& J.E. Vaughan (eds.), Handbook of Set-Theoretic Topology, North-Holland, 1984. MR 85k:54001

11. J.C. Morgan II, Point Set Theory, Marcel Dekker, 1990. MR 91a:54051

12. B.V. Rao, On discrete Borel spaces and projective sets, Bull. Amer. Math. Soc. 75 (1969), 614-617. MR 39:4014

13. B.V. Rao, On discrete Borel spaces, Acta Math. Sci. Hungaricae 22 (1971), 197-198. MR 44:6503

14. R.H. Sorgenfrey, On the topological product of paracompact spaces, Bull. Amer. Math. Soc. 53 (1947), 631-632. MR 8:594f

15. M. Talagrand, Est-ce que $\ell^{\infty}$ est un espace mesurable?, Bull. Sci. Math. 103 (1979), 255-258. MR 80k:03056

Department of Mathematics, Essex University, Colchester C04 3SQ, England

E-mail address: fremdh@essex.ac.uk

Department of Mathematics, Washington State University, Pullman, Washington 99164

E-mail address: johnson@beta.math.wsu.edu

Institute of Mathematics, University of Łódź, S. Banacha 22, 90-238 Łódź, Poland

E-mail address: ewajch@krysia.uni.lodz.pl 